\title{
Política fiscal, mercado de trabajo y empleo informal en México David, Martínez Luis ${ }^{1}$ \\ Universidad Autónoma del Carmen. Ignacio Caamal Cauich \\ Universidad Autónoma Chapingo \\ José Antonio Ávila Dorantes \\ Universidad Autónoma Chapingo. \\ Lucio Alberto Pat Fernández \\ Colegio de la Frontera Sur, Unidad Campeche. \\ (Recibido 11 de enero 2017, aceptado 9 de agosto 2017.)
}

\section{Resumen}

Los cambios en la política económica y el mercado de trabajo pueden influir en la economía informal en México. El artículo tiene como objetivo analizar los efectos de diversas variables macroeconómicas sobre el empleo informal en México. Para determinar estos efectos se diseñó un modelo de ecuaciones simultáneas y se estimó mediante el método de mínimos cuadrados ordinarios en dos etapas. Los resultados indican que el nivel de precios, la producción del sector servicios y el salario mínimo tienen un efecto sobre el empleo informal. Son múltiples los determinantes de la informalidad y es complejo incluirlos en un modelo; sin embargo, el modelo estimado aporta elementos para dirigir la política económica en busca de contrarrestar su crecimiento. Se concluye que un aumento del poder adquisitivo de los salarios formales y la creación de empleos en el sector secundario pueden disminuir el empleo informal. Hasta ahora no se ha estimado un modelo que muestre los factores que afectan el crecimiento del empleo formal e informal.

Clasificación JEL: J46, J21, J31, E23.

Palabras clave: empleo informal, salario minimo, Producto Interno Bruto, elasticidad.

\section{Fiscal policy, labor market and informal employment in Mexico}

\begin{abstract}
Changes in economic policy and the labor market can influence the informal economy in Mexico. The aim of this article is to analyze the effects of a variety of macroeconomic variables on informal employment in Mexico. To determine these effects, a simultaneous equations model was designed and estimated using the two-stage ordinary least squares method. The results indicate that the price levels, production in the service sector, and the minimum wage have an effect on informal employment. There are multiple determinants of informality and it is complex to include them in one model; however, the estimated model provides elements to guide the economic policy in order to counteract its growth. It is concluded that an increase in the purchasing power of formal wages and job creation in the secondary sector may reduce informal employment. Up till now, no model showing the factors affecting growth in formal and informal employment has been estimated.

JEL Classification: J46, J21, J31, E23.

Keywords: informal employment, minimum wage, Gross Domestic Product, elasticity
\end{abstract}

${ }^{1}$ Calle 56 No. 4 Col. Benito Juárez, C.P.24180. Ciudad del Carmen, Campeche 
78 Nueva Época REMEF (The Mexican Journal of Economics and Finance)

\section{Introducción}

En México el crecimiento medio de la productividad del trabajo en los sectores no agropecuarios registró una severa disminución durante la década de los ochenta que se prolongó hasta los primeros años de los noventa (Hernández et al, 2000). La productividad en el sector agrícola no superó el $1.1 \%$ desde el 2003 y el problema se concentró en la producción de granos (FAO, 2013). La baja productividad de la economía se refleja en mayores niveles de marginación, menores salarios y bajas tasas de crecimiento económico (Escalante et al, 2007).

Actualmente, México tiene una fuerte desigualdad del ingreso, no ha disminuido la pobreza de la población (Mayer-Foulkes, 2008, Sheenan y Riosmena, 2013) y tiene una fuerte dependencia económica con Estados Unidos y el resto del mundo (Blecker, 2008). La crisis financiera de 2008 provocó una disminución de la actividad económica, un aumento del desempleo, una disminución de la producción de sectores importantes como manufacturas, construcción y servicios y una disminución de las remesas provenientes del extranjero (Iniguez, 2014).

En el periodo de 1980 al 2015 la tasa de crecimiento real promedio de la economía fue de $2.4 \%$, lo que refleja un estancamiento de la economía mexicana. Para contrarrestar los efectos de las crisis y fomentar crecimiento económico, el gobierno cuenta con la política fiscal (Alcántar-Toledo y Venieris, 2014) y la política monetaria. En lo que respecta a la política fiscal, la recaudación en México es muy baja y el Gobierno Federal no cuenta con los recursos necesarios para generar crecimiento económico a través del gasto público. A pesar de importantes cambios en el sistema tributario como la aparición de nuevos impuestos, aumento de las tasas impositivas, aumento de las bases de contribuyentes y el mejoramiento del sistema de cobro de impuestos, los ingresos del Gobierno Federal sólo representan el $8 \%$ de la Producto Interno Bruto del país, a diferencia de Estados Unidos y Canadá cuyos ingresos son superiores (Martínez, et al., 2009).

La baja recaudación tributaria se debe, entre otros aspectos, a que cerca del $32 \%$ de la economía es informal (Schneider, 2007). De acuerdo con la Encuesta Nacional de Ocupación y Empleo (ENOE) en 2015 existían 13.7 millones de trabajadores informales que representaban el $57.8 \%$ del empleo total (INEGI, 2015).

La economía informal se ha relacionado con el crecimiento real de la economía: a mayor tasa de crecimiento económico menor desempleo y menor proporción de la Población Económicamente Activa (PEA) ocupada en el sector informal (Escribá y Fons, 2014). En el caso de México, la economía informal ha ganado relevancia en los últimos años como resultado de una fuerte inestabilidad del empleo formal durante las crisis económicas (Carr y Chen, 2001) y por el bajo crecimiento económico (Sheenan y Riosmena, 2013). Cuando la política económica no genera crecimiento económico el empleo informal aumenta (Brambila y Cazzabillan, 2009). 
Este trabajo tiene como objetivos analizar los cambios que han sufrido la política fiscal y el mercado de trabajo en México de 1980 a 2015 y determinar mediante un análisis cuantitativo el impacto de estos cambios en el empleo informal. La variable de política fiscal que se estudia es el ingreso tributario. Las variables que están relacionadas con el mercado de trabajo son la tasa de desempleo y el salario mínimo. También se analiza el Índice Nacional de Precios al Consumidor y el PIB terciario como factores que influyen en el empleo informal. Además, se analiza y cuantifica el efecto del empleo informal sobre el Producto Interno Bruto.

En el primer apartado de este artículo se presenta una revisión conceptual de economía informal. En el apartado metodológico se desarrolla un modelo de ecuaciones con tres ecuaciones: en la primera ecuación se toma como variable dependiente al empleo informal y como variables independientes al salario mínimo, los impuestos, la tasa de desempleo, el Índice Nacional de Precios al Consumidor (INPC) y el PIB del sector terciario. En la segunda ecuación se presenta al PIB real en función del empleo informal y el gasto público. En la tercera ecuación se determina que la base monetaria depende del PIB real, del empleo informal y de la inflación.

En el tercer apartado, los resultados se centran en una discusión sobre la influencia de las variables de política económica sobre el empleo informal de 1980 a 2015. La discusión se refuerza con información estadística y con los resultados del modelo de regresión. Finalmente, se plantean las conclusiones del trabajo. Los hallazgos proporcionan evidencia concluyente de que los mercados de futuros de petróleo WTI y Brent son excelentes mecanismos para mitigar la exposición al riesgo de los participantes en los mercados del petróleo mexicano a través de dos modelos dinámicos de correlaciones condicionales. Sin embargo, los resultados del estudio carecen de un sustento estadístico robusto para evaluar el poder predictivo y la precisión de los diferentes modelos GARCH multivariados para la estimación de las RCO fuera de la muestra.

\section{La economía informal, su conceptualización}

La Organización Internacional del Trabajo (OIT) inició con el concepto de sector informal marcando una diferencia entre el trabajo asalariado y el trabajo por cuenta propia (Hart, 1973). Los trabajadores por cuenta propia se caracterizaban por tener baja productividad, bajos ingresos y laboraban sin contratos y sin prestaciones. Además, sus unidades de producción eran pequeñas, con poca tecnología, poco capital y poca organización (Samaniego, 2008).

Bajo este concepto la informalidad fue sinónimo de pobreza, pero no reflejaba la heterogeneidad y la complejidad del fenómeno; por lo tanto, la OIT cambio el término a economía informal. El empleo en la economía informal se denominó "subempleoz se supuso que quienes participaban en él no lograban ingresar a la economía formal (García, 1991; Klein y Tokman, 2000). 
80 Nueva Época REMEF (The Mexican Journal of Economics and Finance)

De Soto (1987) presenta a la economía informal como la respuesta de la población a la incapacidad del Estado de satisfacer las aspiraciones más elementales de la población en pobreza, que sobreviven otorgando el privilegio de participar legalmente en la economía a una pequeña élite. Para De Soto los trabajadores utilizan su costo de oportunidad para elegir trabajar en la economía formal o informal. El costo de oportunidad está determinado por la seguridad social y los derechos laborales a los que renuncia el trabajador por ahorrarse los trámites burocráticos y el pago de impuestos que se imponen en la economía formal. Cuando los trabajadores eligen trabajar en la informalidad se pone de manifiesto que los ingresos obtenidos de las actividades informales llegan a ser superiores a los obtenidos en actividades formales. Así, la economía informal deja de ser sinónimo de pobreza debido a que en muchas ocasiones las actividades informales llegan a ser altamente rentables (Portes y Schauffler, 1993). Es posible acercarse al concepto de economía informal cuando se realiza una clasificación basada en los objetivos que persiguen las actividades informales. Por ejemplo, existe una clara diferencia entre actividades formales, informales e ilícitas (Castells y Portes, 1989). Las actividades formales son aquellas cuyo proceso de producción y el producto final obtenido son completamente lícitos para su comercialización y consumo, mientras que en las actividades ilícitas la producción, la comercialización y el consumo están claramente prohibidas por la ley. Las actividades informales pueden tener un proceso de producción y comercialización ilícito o lícito en el sentido tributario, pero el producto que se genera en estas actividades es completamente lícito.

Para Portes y Haller, (2004) las actividades informales también se pueden clasificar como actividades de supervivencia, de explotación independiente y de crecimiento. En las actividades de supervivencia se ubica la población que realiza actividades con el fin de obtener recursos para sobrevivir a través de la producción y comercialización en el mercado informal. Las actividades de explotación independiente se realizan con la finalidad de reducir los costos de las empresas formales. Las actividades de crecimiento tienen como finalidad la acumulación de capital. Aunque se tienen diferentes objetivos, estos tres grupos pueden estar relacionados; por ejemplo, la población que busca realizar actividades de supervivencia puede estar contribuyendo a la reducción de costos de las empresas formales y una vez que cruce el umbral de supervivencia puede iniciar un proceso de acumulación de capital.

Estas clasificaciones son una muestra de la heterogeneidad y la dinámica de las actividades informales, características que la han llevado a tener diversas terminologías.

Los términos más utilizados para nombrar a las actividades informales son: economía subterránea, economía sumergida, economía gris, economía invisible, economía negra, economía no observada, contra economía; entre otras. A pesar de esta heterogeneidad conceptual, diversos autores consideran que un trabajador informal es aquel que trabaja fuera de las regulaciones del gobierno; 
es decir, se encuentra fuera de las leyes laborales y tributarias (Amaral y Quintin, 2006; Maloney, 1999).

\section{Metodología}

La política económica aplicada en México de 1980 a 2015 ha influido en el crecimiento del empleo informal; para determinar esta relación se diseñó un modelo de ecuaciones simultáneas con base en el modelo de Múltiples Indicadores y Múltiples Causas (MIMIC) propuesto por Schneider (2007). Schneider utilizó el modelo MIMIC para medir el tamaño de la economía informal. En su modelo toma como variable latente a la economía informal; a sus indicadores las variables sobre las cuales se refleja la economía informal y las causas son las variables que afectan su crecimiento. El objetivo de este trabajo es analizar los determinantes del empleo informal y no calcular el tamaño de la economía informal. El empleo informal en México se puede obtener con la diferencia entre el empleo total y los empleos registrados en el Instituto Mexicano del Seguro Social (IMSS) e Instituto de Seguridad y Servicios Sociales de los Trabajadores del Estado (ISSSTE). A partir de lo anterior se puede diseñar el siguiente modelo de ecuaciones simultáneas:

Figura 1. Modelo de causas y efectos del empleo informal.

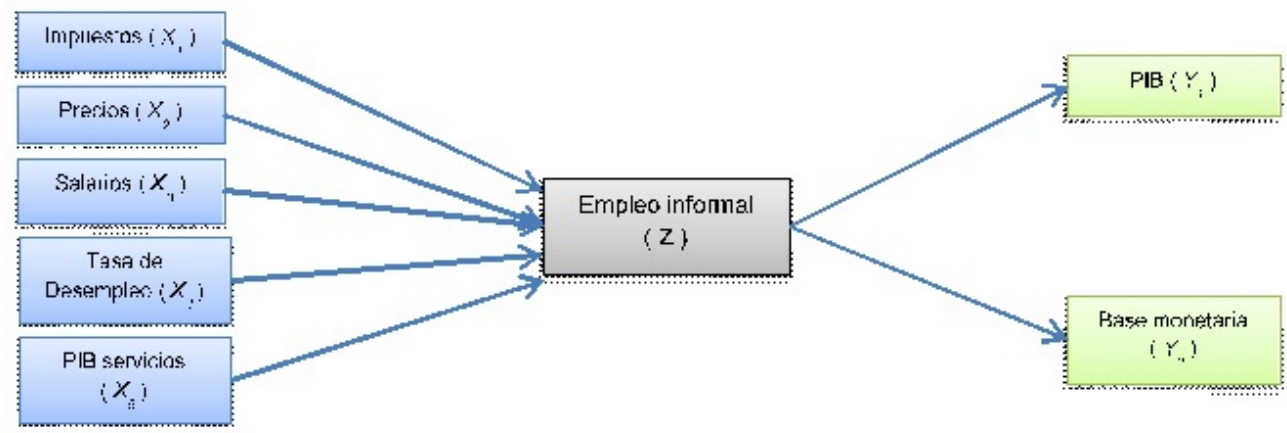

Fuente: Con base en Schneider (2007).

En el modelo propuesto, los indicadores sobre los cuales se refleja el empleo informal son los siguientes:

1. Moneda. El uso de la cantidad de dinero en circulación puede ser un buen indicador del aumento o disminución de las transacciones no registradas. Existen diversos estudios que han demostrado que las actividades 
82 Nueva Época REMEF (The Mexican Journal of Economics and Finance)

informales se reflejan en la demanda de efectivo en una economía (Schneider, 2013; Davidescu, et al, 2015). Nchor (2016) menciona que una fuerte carga impositiva estimula la evasión de impuestos y como consecuencia incrementa la demanda de efectivo. Una parte importante de las transacciones informales tienen lugar utilizando solamente dinero en efectivo para evitar ser detectados (Brambila y Cazzavillan, 2009). Por lo tanto, en este trabajo se utiliza el dinero real en manos del público y se espera que el empleo informal tenga un efecto positivo en la base monetaria (Kolev y Morales, 2005) (Schneider, 2007).

2. El PIB real. Para estimar el empleo informal se necesita una variable de referencia (Kolev y Morales, 2005). Estudios muestran que cerca de las dos terceras partes de los ingresos de los trabajadores informales pasan de inmediato a la economía formal; lo que provoca un efecto positivo en la economía formal (Ernste y Schneider, 1998). Aunado a lo anterior, la economía informal puede contribuir a la creación de mercados, al aumento de recursos financieros y transformar las instituciones legales, sociales y económicas (Ernste y Schneider, 1998)

La política económica influye en el empleo informal a través de las siguientes variables macroeconómicas:

1. Impuestos. Una carga tributaria excesiva incentiva la evasión de impuestos (Torgler y Schneider, 2009; Kolev y Morales; 2005; Schneider, 2000; Johnson, et al, 1998) y empuja a los agentes económicos a utilizar mano de obra informal (Farell, 2004). La informalidad laboral permite eludir contribuciones patronales de empresas e instituciones privadas y públicas (Bonilla, 2015). Cuando los mercados tienen menos regulaciones la informalidad y el desempleo disminuyen; y mientras los impuestos son mayores en el sector formal el tamaño relativo del sector informal aumenta (Charlot, et al, 2014). Aunado a lo anterior, los impuestos laborales disminuyen el salario real de los trabajadores haciendo más atractivo ingresar al sector informal (Araujo y Rodrigues, 2016).

2. Nivel de precios. Las políticas que disminuyen la tasa de interés y promueven el acceso al crédito fomentan la creación y fortalecimiento de las pequeñas y medianas empresas y es posible reducir la informalidad (Rodríguez y Calderón, 2015). Altas tasas de inflación en una economía provocan un incremento de la tasa de interés y el sector crediticio se desestabiliza, lo cual acaba con las pequeñas empresas y se fomenta el mercado negro (Koreshkova, 2006). Las altas tasas de inflación también se reflejan en una disminución de los salarios reales y alientan a la población a buscar otras fuentes de ingreso en la economía informal (Gunther y Launov, 2012).

3. Salarios: El trabajo informal aparece como alternativa cuando se presenta una disminución del ingreso familiar (Brambila y Cazzavillan, 2009; 
Gunther y Launov, 2011). Los salarios en México han perdido poder adquisitivo desde hace más de 30 años. El salario mínimo ha crecido a tasas por debajo de la inflación de 1980 a 2015, lo que permite a las empresas establecidas aprovecharse de los trabajadores no calificados, que se ven incentivados a buscar mejores oportunidades y mejores fuentes de ingresos dentro de la informalidad (Maloney, 1999).

4. Desempleo: Diversos autores han concluido que el crecimiento del empleo informal se debe a la falta de capacidad del sector formal de generar empleos (Cortés, 2000; Tokman, 2001). Si el trabajo en el sector formal es escaso, la población tiende a buscar soluciones a su falta de ingreso mediante la venta o prestación de servicios que les permita sobrevivir (Samaniego, 2008). Un aumento del desempleo debe alentar a los trabajadores despedidos a intentar fortuna en los mercados informales (Gúnther y Launov, 2012; Schneider y Enste, 1998).

5. Sector terciario. El crecimiento urbano y la tercerización de la economía fomentan el empleo informal (Campos e Iglesias, 2002). La población que emigra del campo a la ciudad y que no encuentra empleo en la industria ofrecen su trabajo de baja productividad en el sector servicios informal (Ramos, 2015). El crecimiento del sector servicios de la economía ha provocado una mayor utilización de mano de obra no calificada (Montes-Rojas y Santamaria, 2007) y barata, porque la población prefiere buscar empleos en actividades informales mejor remuneradas (Leal, 2013). El crecimiento desproporcionado del sector terciario se refleja en una economía caracterizada por una fuerte informalidad (Aguilar, 2006).

A partir de los indicadores y causas antes mencionadas se puede establecer el siguiente sistema de ecuaciones simultáneas.

$$
\begin{gathered}
Z=\alpha_{0}+\alpha_{1} X_{1}+\alpha_{2} X_{2}+\alpha_{3} X_{3}+\alpha_{4} X_{4}+\alpha_{5} X_{5} \cdots \\
Y_{1}=\beta_{0}+\beta_{1} Z+\beta_{2} X_{6} \cdots \\
Y_{2}=\Theta_{0}+\Theta_{1} Z+\Theta_{2} Y_{1}+\Theta_{3} X_{7} \ldots
\end{gathered}
$$

Donde:

$Z=$ Empleo informal en México

$X_{1}=$ Impuestos en México

$X_{2}=$ Índice Nacional de Precios al Consumidor 
84 Nueva Época REMEF (The Mexican Journal of Economics and Finance)

$X_{3}=$ Salario Mínimo en México

$X_{4}=$ Tasa de desempleo en México

$X_{5}=$ PIB terciario en México

$Y_{1}=$ PIB real de México

$X_{6}=$ Gasto público en México

$Y_{2}=$ Base Monetaria en México

$X_{7}=$ Inflación en México

$\alpha i, \beta i, \Theta i=$ Parámetros a estimar

El modelo anterior permite cuantificar el efecto de las principales variables de política económica sobre el empleo informal; cuantificar el impacto del empleo informal y el gasto público en la producción; y cuantificar el impacto de la producción, el empleo informal y la inflación en la cantidad de dinero en circulación en la economía. La estimación del modelo se realizó mediante el método de Mínimos Cuadrados en Dos Etapas. Debido a la falta de datos oficiales, el modelo se estimó con datos de 1980 a 2010.

\section{Resultados y Discusión}

\subsection{Factores macroeconómicos y el empleo informal en México}

La política fiscal se relaciona con el empleo informal a través del cobro de impuestos. En la economía informal se generan incentivos para la evasión fiscal y existe evidencia de que los individuos participan en este sector en la búsqueda de evadir las cargas fiscales (Ernste y Schneider, 1998). En muchos casos, los individuos aumentan su utilidad al incorporarse al sector informal, ya que en éste las posibilidades de evasión fiscal o de incumplimiento de las normas laborales son mayores (Núñez, 2002).

En México se han realizado diversas reformas fiscales para fortalecer los ingresos públicos y disminuir el sector informal. En los noventa aumentó el Impuesto Sobre la Renta (ISR), aumentó el Impuesto al Valor Agregado (IVA) y el Sistema de Administración Tributaria (SAT) simplificó muchos trámites para el pago de impuestos. A pesar de estas reformas, a finales de esta década cerca de 10 millones de trabajadores no pagaban impuestos, la recaudación fiscal fue muy baja y aumentó la actividad informal (Chávez, 2001).

La reforma fiscal de 2007 se centró en combatir la informalidad a través del Impuesto a Depósitos en Efectivo (IDE). Con el IDE se cobró el $2 \%$ de los depósitos bancarios en efectivo mayores a 25 mil pesos. En 2009 se aprobó un aumento del IVA de $15 \%$ a $16 \%$, y debido a que aumentó la informalidad, el IDE se cobró al $3 \%$ sobre los depósitos mayores a 15 mil pesos. En 2014 desaparece el IDE y al mismo tiempo aumenta la tasa máxima de ISR de 30 a $35 \%$.

La desaparición del IDE es una muestra de que las reformas implementadas no han contribuido a disminuir la informalidad; por el contrario, el pago de impuestos se concentra en los contribuyentes cautivos restando competitividad a los salarios del trabajador formal. El aumento del IVA y la aparición de nuevos impuestos como el gravamen a telecomunicaciones, gravamen a bebidas y 
alimentos "chatarras", gravamen a alimentos para mascotas, entre otros, disminuyen el poder adquisitivo de los salarios formales e informales. Los aumentos en el ISR afectan directamente a los salarios reales del sector formal.

\subsection{Los salarios reales y la economía informal}

En los países en desarrollo el salario mínimo determina el empleo y los salarios en el sector formal y produce un efecto "faro" en el sector informal. En algunos casos el salario mínimo desincentiva el empleo informal sobre todo en los trabajadores que perciben ingresos cercanos al salario mínimo (Maloney y Núnez; 2004). Los salarios promedio en el sector formal son superiores a los del sector informal en muchos países; sin embargo, no existe consenso acerca de las causas de esta brecha salarial (Günther y Launov, 2012). Se ha observado que los trabajadores formales tienen más experiencia, más educación y ganan más que los trabajadores informales. Estos hechos prueban que los trabajadores poco cualificados enfrentan barreras para entrar al sector formal; sin embargo, existe poca evidencia de que tales barreras son importantes (Amaral y Quintin, 2005).

En México los deficientes servicios públicos, que forman parte de la protección social, y los bajos niveles de productividad del trabajo hacen que el empleo del sector informal sea una alternativa de generación de ingresos y de mejoramiento de la calidad de vida de algunos sectores de la población (Maloney, 1999). Al comparar los salarios y el tipo de trabajo en el sector formal e informal se pone en duda que el sector informal sea un refugio para la población desempleada y que los trabajadores informales son mal pagados (Marcouiller, et al, 1995).

Independientemente de las diferencias salariales entre el sector formal e informal, las actividades informales se han convertido en una opción para generar mayores ingresos para la población (Wallace y Latcheva, 2006); sobre todo cuando el poder adquisitivo de los salarios en el sector formal se ve mermado por el cobro de impuestos y la inflación.

\subsection{La inflación y la economía informal}

A pesar de que el control de precios fue uno de los objetivos de la política económica planteada a inicios de los ochenta, la inflación en 1986 fue de $106 \%$ y en 1987 fue de $159 \%$. Con la firma del Pacto de Solidaridad Económica en 1987, inició un descenso inflacionario influenciado por las políticas de ajuste en las finanzas públicas, el establecimiento de un tipo de cambio fijo, el control de la expansión de la cantidad de dinero y la liberalización comercial (Noyola, 2009). El descenso de la inflación en los años noventa y la estabilización de los últimos años se fundamentan en un control estricto de la oferta monetaria y en la contracción de los salarios reales. 
86 Nueva Época REMEF (The Mexican Journal of Economics and Finance)

Gráfica 1. Evolución del salario mínimo real en México (Pesos a precios de 2011).

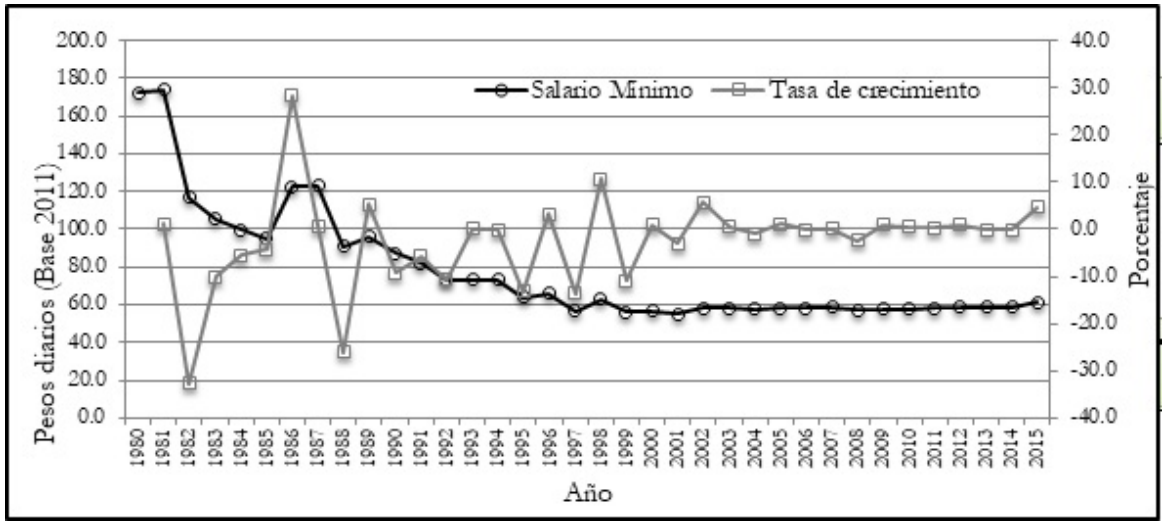

Fuente: con base en datos de la Secretaría de Trabajo y Previsión Social.

La inflación afecta al mercado de trabajo a través del salario real. En México, el salario mínimo real disminuyó en aproximadamente $60 \%$ de 1980 a 2015 (Gráfica 1). La disminución del poder adquisitivo de los salarios provocó que otros integrantes de las familias ingresaran al mercado de trabajo en busca de mejorar sus ingresos. Las mujeres y los jóvenes aumentaron su participación en la PEA y aumentaron la oferta de empleos de medio tiempo y mal remunerados (Pacheco y Parker, 2001). Este fenómeno, aunado a la política de estabilidad de precios basado en la reducción de la inercia salarial, disminuyó la presión del salario nominal y el salario mínimo creció a tasas inferiores a la inflación.

\subsection{El mercado de trabajo y el empleo informal en México}

El crecimiento de la informalidad también se explica por las características del mercado de trabajo (Marjit et al, 2004). En países que tienen una alta tasa de desempleo, bajos salarios y fuertes cargas fiscales la economía informal tiende a crecer (Brambila y Cazzavillan, 2009). La economía informal surge porque existe un sector de la población que no puede trabajar o iniciar negocios de manera formal (Ochoa, 2002) y recurre a la economía informal como una opción para generar ingresos. En México, la tasa de desempleo abierto es baja comparada con países como Estados Unidos y Canadá. Sin embargo, la economía informal y la migración internacional han tenido un crecimiento constante en los últimos años. Por ejemplo, en el año 2000 más de 7 millones de mexicanos residían en Estados Unidos y el saldo neto de migración fue de alrededor de 300 mil trabajadores por año (Hernández, 2000). El crecimiento de la economía informal y de la migración internacional pone en duda que se estén generando empleos formales en el país.

Un análisis de la tasa de desempleo abierto en México muestra que en 
las últimas dos décadas entre el $35 \%$ y $40 \%$ de la PEA desocupada se concentraba en la población con secundaria y entre el $30 \%$ y $40 \%$ en la población con educación media superior y superior. Al sumar estos dos grupos se observa que más del $50 \%$ del desempleo se concentra en la población con secundaria y con estudios de educación media superior y superior. Los empleos que se generan en México no son de calidad; por el contrario, reflejan que el sector productivo del país utiliza mano de obra poco cualificada y con baja productividad. De acuerdo con la Encuesta Nacional de Ocupación y Empleo (ENOE), el desempleo se refleja en mayor proporción en los jóvenes, ya que la población entre 14 y 29 años de edad que están desempleados y que además no trabajan ni estudian es de alrededor de 7.5 millones, lo que representa el $16 \%$ de la PEA.

Cuadro 1. Distribución de la población desempleada abierta por nivel de instrucción (porcentaje).

\begin{tabular}{|l|l|l|l|l|}
\hline Año & $\begin{array}{l}\text { Primaria in- } \\
\text { completa }\end{array}$ & $\begin{array}{l}\text { Primaria } \\
\text { completa }\end{array}$ & $\begin{array}{l}\text { secundaria } \\
\text { completa e } \\
\text { incompleta }\end{array}$ & $\begin{array}{l}\text { Medio supe- } \\
\text { rior y supe- } \\
\text { rior }\end{array}$ \\
\hline 1990 & 8.9 & 18.6 & 43.9 & 28.6 \\
\hline 1995 & 11.5 & 17.4 & 40.0 & 31.1 \\
\hline 2000 & 6.2 & 13.1 & 36.9 & 43.8 \\
\hline 2005 & 11.5 & 20.7 & 36.7 & 31.1 \\
\hline 2010 & 9.0 & 18.8 & 37.7 & 34.4 \\
\hline 2015 & 6.7 & 16.0 & 39.9 & 37.4 \\
\hline
\end{tabular}

Fuente: INEGI. Encuesta Nacional de Ocupación y Empleo. Varios años.

Gráfica 2. Población ocupada por nivel de ingreso en 2015 en México (Porcentaje).

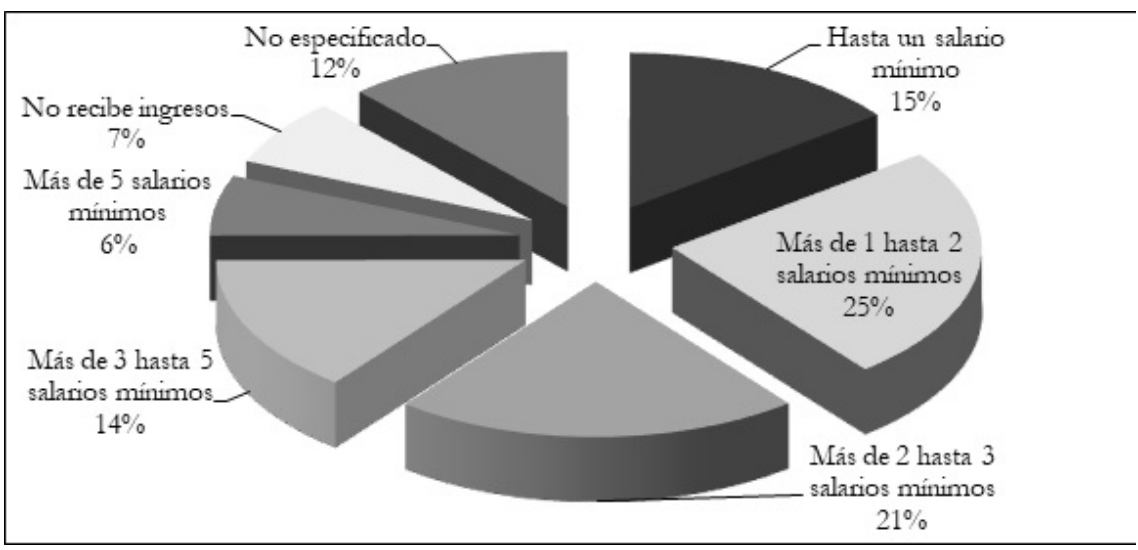

Fuente: INEGI. Encuesta Nacional de Empleo, 2015. 
88 Nueva Época REMEF (The Mexican Journal of Economics and Finance)

La terciarización de la economía es una causa de la falta de empleos de calidad. Una parte importante de los empleos generados en el sector servicios no requiere de una fuerte especialización (Carr y Chen; 2001). Como resultado, el $61 \%$ de la población ocupada gana menos de 3 salarios mínimos (200 pesos diarios), mientras que la población ocupada que gana más de 5 salarios mínimos representó únicamente el $6 \%$ del total en 2015 (INEGI, 2016). Los empleos generados son mal pagados y por ello los salarios se han convertido en un factor importante del crecimiento de la migración internacional y de la economía informal.

En la mayoría de los países en desarrollo se han implementado políticas de liberalización comercial que han ocasionado un incremento del sector informal y un aumento del desempleo (Chauduri y Banerjee, 2007). La industria mexicana ha sido afectada por la desactivación y restructuración de la economía y por el cambio de política económica hacia el exterior. En México existe una marcada tercerización de la fuerza de trabajo como resultado de una contracción del empleo industrial; aunque en los ochenta y noventa se incrementaron considerablemente las exportaciones manufactureras, la actividad industrial ha perdido importancia en la generación de empleos en el país (Gráfica 3). Para algunos autores, esta situación se presenta en la economía informal ya que los empleos informales en el sector secundario se trasladan al sector terciario en algunos países (Amadeo y Pero, 2000). Además, el sector terciario informal también absorbe la mano de obra desplazada del sector agropecuario (Tokman, 1982).

Gráfica 3. Personal ocupado por actividad económica en México (Miles de personas).

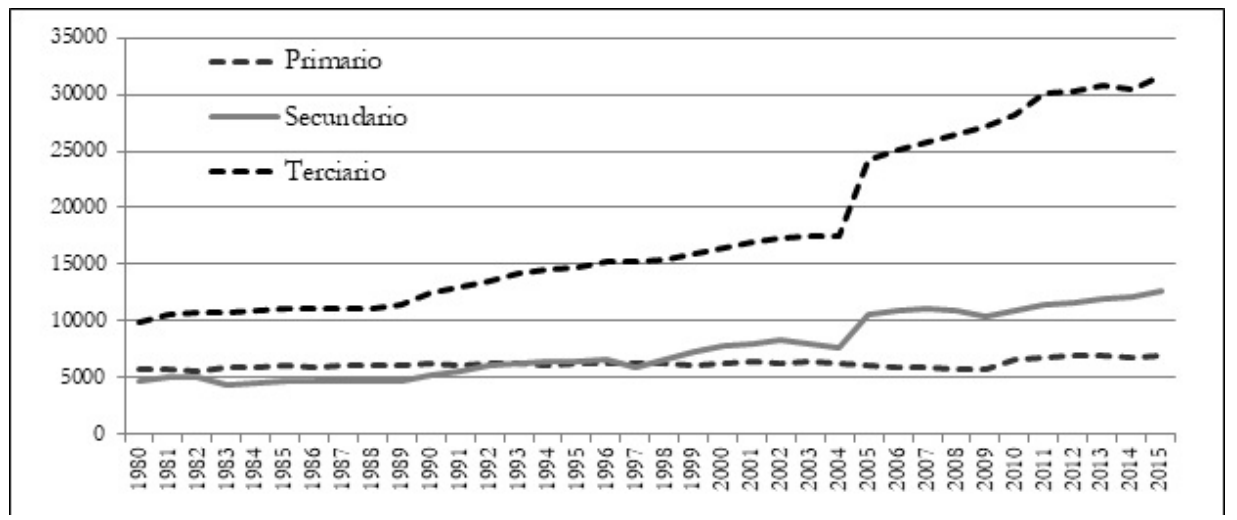

Fuente: Instituto Nacional de Estadística, Geografía e Informática. INEGI. www.inegi.org.mx

Existe evidencia que expone a los trabajadores del sector informal en condiciones menos adversas. Una gran cantidad de trabajadores formales deciden emplearse por cuenta propia y encuentran en el ámbito informal un espacio de alta rentabilidad para sus actividades productivas, aun cuando la estabilidad 
del empleo formal es más valorada que tener mejores salarios (Castro y Huesca, 2007). Este fenómeno se profundiza debido a que en los últimos años el sector formal genera más empleos eventuales que permanentes (Gráfica 4).

Para la población es relativamente fácil entrar a la informalidad (Günter y Launov, 2009) debido a que en este sector se requieren de bajos niveles de educación, poca tecnología y poco capital (Sheenan y Riosmena, 2013). El sector informal comprende una diversidad de actividades y varios tipos de relaciones laborales como el trabajo por cuenta propia en actividades de subsistencia (venta ambulante, recolección de basura y otros); el trabajo a domicilio en régimen de tercerización; o el trabajo independiente en microempresas individuales (Brambila y Cazzabillan, 2009).

Gráfica 4. Tasa de crecimiento del empleo formal en México (Porcentaje).

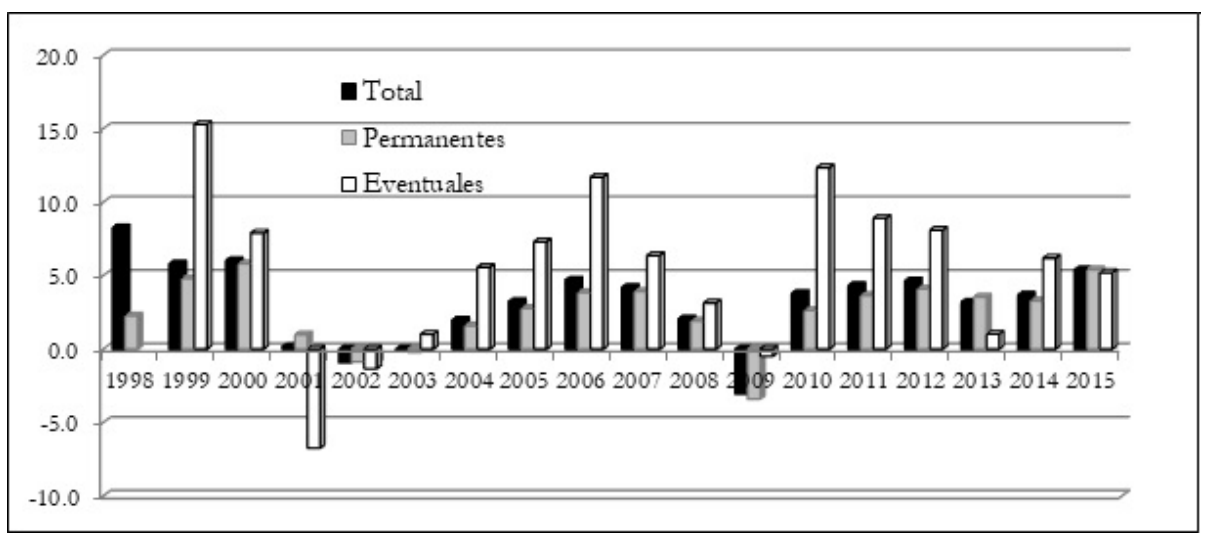

Fuente: Elaborado con datos del Instituto Mexicano del Seguro Social.

El análisis anterior muestra como las políticas económicas han impulsado el crecimiento del empleo informal; hoy en día la economía informal es la principal fuente de empleo en México y constituye un desperdicio de recursos que merman la capacidad productiva del país (Brambilla y Cazavilla, 2009). El gran volumen del sector informal disminuye la capacidad real del Estado para abarcar con sus instrumentos a toda la estructura del país, mermando al mismo tiempo los ingresos públicos y repercutiendo en la calidad de los servicios que el Estado ofrece. Es necesario reformular la política en general, para llegar a una política de empleo que lleve al país a generar empleos formales e incorporar empleos informales a la formalidad (Samaniego, 2008).

\section{Resultados del modelo de regresión}

Los resultados de la ecuación de empleo informal indican que existe una relación inversa entre el ingreso tributario y el empleo informal; un aumento del ingreso tributario en $1 \%$ genera una disminución del empleo informal de $0.09 \%$. 
90 Nueva Época REMEF (The Mexican Journal of Economics and Finance)

Dentro de los ingresos tributarios el ISR y el IVA representan cerca del $90 \%$; aunque el efecto de estos impuestos sobre el empleo informal es débil; es claro que guardan una relación directa (Schneider y Enste, 2009; Neck, et al, 1989; Loayza, 1996, charlot, et al, 2014; Araujo y Rodrigues, 2016).

La política económica en los ochenta se centró en lograr la estabilidad de precios a través de la contracción de los salarios reales. La política de control de precios en México se reflejó en un aumento del empleo informal debido a que parte de la población se refugió en la informalidad para buscar otras fuentes de ingreso (Gunther y Launov, 2012). Aunado a lo anterior, las altas tasas de inflación se reflejaron en las tasas de interés y el acceso al crédito de las pequeñas empresas fue más difícil y se fomentó el mercado informal (Koreshkova, 2006). El resultado fue un nulo crecimiento del salario mínimo y el crecimiento del empleo informal. Los resultados del modelo confirman los planteamientos anteriores debido a que en México un incremento del INPC en $1 \%$ provoca un crecimiento del empleo informal de 0.32

El salario mínimo guarda una relación indirecta con el empleo informal; al aumentar el salario mínimo en México en $1 \%$ el empleo informal disminuye en $0.29 \%$; por lo que existe una relación inversa entre estas variables (Brambila y Cazavillan, 2009; Gunther y Launov, 2011). La relación encontrada entre el salario mínimo y el empleo informal es inelástica; pero de todas las variables analizadas es una de las que más influyen en el empleo informal. En México, el salario mínimo aumentó a tasas inferiores a la inflación perdiendo poder de compra de 1980 a 2015. La reducción de su ingreso real obligó a la población a buscar otras fuentes de ingresos en mercados más flexibles como el informal (Wallace y Latcheva, 2006; Brambila y Cazzavillan, 2009; Gunther y Launov, 2011).

En México, a partir de 1994 se abrió la brecha entre el empleo terciario y el empleo secundario; lo anterior muestra un crecimiento desproporcionado del sector servicios (gráfica 3) y aunado a la fuerte migración del campo a la ciudad, gran parte del trabajo informal se ha concentrado en este sector (Aguilar, 2006). El crecimiento del sector servicios tiene un efecto directo sobre el empleo informal; cuando la producción del sector servicios aumenta en $1 \%$ el empleo informal aumenta en $0.17 \%$.

No se puede establecer una relación entre el desempleo y el empleo informal debido a que el coeficiente obtenido en el modelo de regresión no es estadísticamente significativo. Cuando la economía informal es muy grande muchos indicadores económicos no son reales y es muy difícil encontrar los efectos de las políticas en la economía (Schneider y Enste, 2000). Al respecto, la tasa de desempleo en México se calcula independientemente si la población labora en la economía formal o informal; además, tampoco considera la cantidad de horas laborales. Por lo anterior, no se puede observar una relación entre el desempleo y el empleo informal con las estadísticas oficiales. Schneider y Enste (2000) mencionan que cuando disminuyen las horas trabajadas en el sector formal la informalidad aumenta. En México este fenómeno se podría observar con la Tasa 
de Desocupación Parcial que no fue utilizada en este trabajo.

Cuadro 2. Estimadores y elasticidades de la función de empleo informal, producción y base monetaria.

\begin{tabular}{|c|c|c|c|c|}
\hline Ecuación & $\begin{array}{c}\text { Variables } \\
\text { independientes }\end{array}$ & $\begin{array}{c}\text { Estimadores } \\
\text { de } \\
\text { parámetros }\end{array}$ & $\operatorname{Pr}>|t|$ & Elasticidad \\
\hline \multirow[t]{6}{*}{$\begin{array}{c}\text { Ecuación de } \\
\text { Empleo } \\
\text { Informal }\end{array}$} & & - & & \\
\hline & Ingreso tributario & -3.2 & 0.0373 & -0.09 \\
\hline & Nivel de precios & $102,200.0$ & 0.0108 & 0.32 \\
\hline & Salario mínimo & $-176,139.0$ & 0.0126 & -0.29 \\
\hline & Desempleo & $173,521.9$ & 0.0550 & 0.06 \\
\hline & PIB servicios & 0.6 & 0.0196 & 0.17 \\
\hline $\mathrm{Fcal}=4.76$ & $\begin{array}{l}\mathrm{Pr}>\mathrm{F}=0.0039 \\
\mathrm{R}^{2}=0.5084\end{array}$ & & & \\
\hline \multicolumn{5}{|l|}{$\begin{array}{l}\text { Ecuación de } \\
\text { producción } \\
\text { real }\end{array}$} \\
\hline & Empleo informal & 883.5 & 0.0168 & 1.85 \\
\hline $\mathrm{Fcal}=111.74$ & $\begin{array}{l}\text { Gasto real } \\
\mathrm{Pr}>\mathrm{F}=0.0001 \\
\mathrm{R}^{2}=0.8857\end{array}$ & $3,958.0$ & 0.0001 & 0.42 \\
\hline \multicolumn{5}{|l|}{$\begin{array}{c}\text { Ecuación de } \\
\text { Base } \\
\text { monetaria }\end{array}$} \\
\hline & Empleo informal & -0.1 & 0.1181 & -5.44 \\
\hline & PIB real & 0.0 & 0.0001 & 4.59 \\
\hline & Inflación & $2,337.3$ & 0.0013 & 0.41 \\
\hline $\mathrm{Fcal}=46.56$ & $\begin{array}{l}\mathrm{Pr}>\mathrm{F}=0.0001 \\
\mathrm{R}^{2}=0.8481\end{array}$ & & & \\
\hline
\end{tabular}

Fuente:Elaboración propia.

La ecuación de la producción real permite observar la importancia del empleo formal y el gasto público en la economía. El gasto público tiene una relación inelástica con el PIB nacional y este efecto es menor al causado por el empleo informal. La magnitud de informalidad a la que se enfrenta la economía se refleja en la baja efectividad de la política de gasto sobre el crecimiento económico.

Los resultados indican que existe una relación directa entre el empleo informal y la producción nacional, por lo que un aumento del empleo informal en $1 \%$ produce un aumento de la producción nacional de $1.85 \%$. 
La magnitud del sector informal está influyendo en el crecimiento del sector formal de la economía mexicana (Markus, Adam y Ginsburg, 1985). Schneider y Enste (2000) encuentran que una proporción de los ingresos obtenidos en la economía informal son utilizados en la economía formal. En México el $59 \%$ de los trabajadores obtienen ingresos del sector informal. Muchos productos que consumen los trabajadores informales se adquieren en supermercados, tiendas comerciales, mercados locales y tiendas de conveniencia fomentando la actividad formal. De acuerdo con algunos cálculos, más del $60 \%$ del ingreso generado por los trabajadores informales se distribuye en los comercios formales (Ernste y Schneider, 1998).

El crecimiento de la economía informal también incentiva la producción del país debido a que algunas empresas formales pueden obtener insumos de esta economía. Las empresas formales utilizan mano de obra informal subcontratada con el objetivo de evadir impuestos, reducir sus costos de producción, aumentar su rentabilidad y aumentar su competitividad (Parra, 2013; Portes, 1995).

Los resultados indican que se ha formado un círculo vicioso en torno a la economía y el empleo formal. En países en desarrollo con una fuerte cantidad de trabajadores informales los ingresos del estado son muy bajos; lo que a su vez reduce la calidad y cantidad de servicios públicos. La informalidad fomenta el aumento de las tasas impositivas y el deterioro de los servicios públicos; por lo tanto, se generan más incentivos para participar en la economía informal (Schneider y Enste, 2000).

El acceso de los trabajadores a servicios sociales y prestaciones laborales son un incentivo para que la población labore en el sector formal. En México no se generan esos incentivos debido a que algunos servicios públicos y de seguridad social son deficientes. Además, los trabajadores formales e informales pueden tener acceso a servicios públicos como educación y salud otorgados por el Estado; por lo que éstos no son determinantes en la disminución del empleo informal (Loaiza, 1996; Schneider y Enste, 2000; Peter de Gijsel, 1984). El bajo crecimiento económico y la fuerte economía informal, ha provocado que la economía formal no funcione como motor en contra de la informalidad ya que la población cuenta con mejores beneficios en la informalidad.

Finalmente, la ecuación de la base monetaria muestra el efecto del empleo informal, el PIB real y la inflación en la cantidad de dinero que hay en circulación. No se puede establecer una relación entre el empleo informal y el circulante en la economía como lo han encontrado Kolev y Morales, (2005) y Schneider, (2007), debido a que el coeficiente obtenido no es estadísticamente significativo. La producción real tiene una relación directa con la cantidad de dinero en la economía, es decir; si el PIB aumenta en $1 \%$ el circulante de la economía aumenta en $4.59 \%$. La inflación tiene una relación directa con la cantidad de dinero en circulación; si la inflación aumenta en $1 \%$ la base monetaria aumenta en $0.41 \%$. 


\section{Conclusiones}

La concentración de la carga fiscal en los contribuyentes cautivos y el crecimiento de las tasas impositivas vuelven menos rentable a las actividades formales. La PEA tiende a elegir contar con un mayor ingreso en el sector informal. La baja recaudación fiscal, derivado una fuerte informalidad, se refleja en el deterioro de los servicios públicos y de la seguridad social y no se generan incentivos para emplearse en el sector formal. Además, los trabajadores informales pueden acceder a servicios públicos y seguridad social (acceso al seguro popular) y las ventajas de tener un empleo formal se están perdiendo.

La pérdida del poder adquisitivo de los salarios ha provocado que el empleo formal pierda competitividad ante los empleos informales. Por lo tanto, las políticas que tienen el efecto de contraer el salario nominal y real, disminuyen el poder adquisitivo de los trabajadores y fomentan las actividades informales.

El coeficiente obtenido entre el desempleo y el empleo informal no es estadísticamente significativo. Lo anterior se explica porque la tasa de desempleo abierto que se reporta es muy baja y no refleja las condiciones reales del mercado de trabajo formal. En realidad, una parte importante de la PEA cuenta con empleos menores a 8 horas diarias; y los empleos eventuales en el sector formal crecen a tasas mayores que los empleos permanentes, son mal remunerados y no son especializados. Aunado a lo anterior, el desempleo en México se concentra en la PEA con mayor nivel de escolaridad y menor a 29 años, lo que refleja la precariedad de los empleos formales en el país. Es así que el crecimiento continuo de la economía informal proporciona indicadores económicos incorrectos como la tasa de desempleo en México.

La importancia que tiene el sector terciario en la generación de empleos es resultado de la política exterior del país, la cual coloca a la industria nacional en competencia internacional y en donde únicamente las empresas más productivas pueden competir. La baja productividad de la industria nacional y su poca capacidad de generar empleos permanentes y bien remunerados, alientan a la fuerza de trabajo a buscar empleo en el sector informal, donde son mal pagados y poco productivos.

El empleo informal tiene un fuerte efecto en el crecimiento económico al tener una relación elástica. Esto se explica porque más de la mitad de los empleos en México son informales y las familias que dependen del empleo informal gastan su ingreso no solamente en la economía informal, sino también en la economía formal. La población en México carece de incentivos para entrar a la economía formal; por ello es indispensable contar con un sistema de seguridad social y servicios públicos que funcionen como incentivo para disminuir el empleo informal.

En la actualidad, en México se ha creado un círculo vicioso, la fuerte presencia de la economía informal reduce los ingresos tributarios debilitando la efectividad de la política fiscal en la búsqueda de crecimiento económico. La falta de crecimiento económico y la precariedad de los empleos formales han generado un crecimiento del empleo informal a tal magnitud, que los trabajadores 
94 Nueva Época REMEF (The Mexican Journal of Economics and Finance)

informales ahora impulsan a la economía formal.

Para contrarrestar el empleo informal en México es necesario que se amplié aún más la base de contribuyentes para quitarle presión al aumento de los impuestos y al mismo tiempo elevar los ingresos tributarios. Un aumento de los ingresos tributarios puede impulsar al crecimiento económico y al mismo tiempo mejorar los servicios públicos y la seguridad social en el país. Para que la economía formal se atractiva también es necesario aumentar los salarios nominales formales que, aunado a una política de estabilización de precios, permita mejorar el poder adquisitivo de los trabajadores formales. Las medidas anteriores deben estar acompañadas de una política industrial que genere un mayor crecimiento del sector secundario, para que sea capaz de crear empleos especializados y mejor remunerados.

\section{Bibliografía}

Aguilar, A. G. (2006). Reestructuración económica y costo social en la Ciudad de México. Una metrópoli periférica en la escala global. Estudios Urbanos contemporáneos. UNAMIIEC/Miguel Ángel Porrúa, México. Pp. 125-166.

Alcántar-Toledo, J. y Y. Venieris. (2014).Fiscal policy, growth, income distribution and sociopolitical instability. European Journal of Political Economy, 34, pp. 315-331.

Amadeo, E. y V. Pero. (2000). Adjustment, Stabilization and the Structure of Employment in Brazil. Journal of Development Studies, 36(4), pp. 120-148.

Amaral, P. y E. Quintin. (2006). A competitive model of the informal sector. Journal of Monetary Economics, 53(7), pp. 1541-1553.

Araujo, J. P. y M. Rodrigues. (2016). Taxation, credit constraints and the informal economy. Economia, (17), pp. 43-55.

Blecker, R. (2008). External shocks, structural change, and economic growth in Mexico, 19792007. World Development, 37( 7), pp. 1274-1284.

Brambila, J. y G. Cazzavillan. (2009). The dynamics of parallel economies. Measuring the informal sector in Mexico. Research in Economics, 63(3), pp. 188-199.

Campos, J. E. y N. Iglesias. (2002). Informalidad y (sub)desarrollo. Ediciones Cal y Arena. México.

Carr, M. y M. Cheen. (2001). Globalization and the informal economy: how global trade and investment impact on the working poor. ILO, pp. 1-29. Disponible en http://www.ilo.org/public/libdoc/ilo/2002/102B09 $55_{\text {engl.pdf }}$

Castro, D. y L. Huesca. (2007). Desigualdad salarial en México: una revisión. Papeles de población, (54), pp. 225-264.

Charlot, O., F. Malherbet y C. Terra. Imformality in deloping economies: Resgulation and fiscal policies. Journal of Economic Dynamic Control, (51), pp. 1-27.

Chaudhuri, S. y D. Banerjee. (2007). Economic liberalization, capital mobility and informal wage in a small open economy: A theoretical analysis. Sciencie Direct, Economic Modelling, 24(6), pp. 924-940.

Chávez, M. (2001). El fracaso de las políticas de estabilización en México: Retos y opciones de política económica. Programa sobre ciencia, tecnología y desarrollo. Documento de trabajo, el Colegio de México, 1(4), pp. 1-32.

Cortés, F. (2000). La metamorfosis de los marginales: la polémica sobre el sector informal en América Latina. Tratado Latinoamericano de sociología del trabajo. Fondo de Cultura Económica, México, pp. 592-618. México.

Davidescu, A., V. A. Strat y A. M. Paul. (2015). Revisting the size of romanian informal economy using the Gutmann approach. Procedia, Economics and Financie (23), pp 10371045. 
De Soto, H. (1987). El otro sendero. Editorial la Oveja Negra. Bogotá, Colombia.

Ernste, D. y S. Friedich. (1998). Increasing shadows economies all over the world - fiction or reality?. IZA DP Discussion Paper Series, (26), pp. 1-65.

Escribà, V. y J. Fons. (2014). Crisis económica y condiciones de empleo: diferencias de género y respuesta de las políticas sociales de empleo. Gaceta Sanitaria, 28(1), pp. 37-43.

Farrel, D. (2004). The hidden dangers of the informal economy. The McKinsey Quarterly, (3), pp. 26-37.

FAO. (2000). El estado mundial de la agricultura y la alimentación. Colección FAO. Roma.

García, N. 1991. Reestructuración, ahorro, y mercado de trabajo. 1a ed., PREALC, Santiago, Chile.

Günther, I. y A. Launov. (2012). Informal employment in developing countries opportunity or last resort?. Journal of Developmet Economics, 97(1), pp. 88-98.

Hart, K. (1973). Informal income opportunities and urban employment in Ghana. Journal of Modern African Studies, 11(1), pp. 61-89.

Hernández, E. (2000). Productividad y empleo en la apertura económica de México. Revista el Trimestre Económico, 67 (265), pp. 121-153. México.

Hernández, E., N. Garro e I. Llamas. (2000). Productividad y mercado de trabajo en México. Plaza y Valdes Editores. Primera edición. México.

INEGI. (2015). Encuesta Nacional de Ocupación y Empleo. INEGI. Aguascalientes, México.

Iniguez, A. J. (2014). Growth with equity for the development of Mexico: poverty, inequality, and economic growth (1992-2008). World development, 59, pp. 313 - 326.

Johnson, S., D. Kaufmann y P. Zoido-Lobatón. (1998). Regulatory discretion and the unofficial economy. American Economy Rewiev, 88(2), pp. 387-392.

Klein, E. y V. E. Tokman. 2000. La estratificación social bajo tensión en la era de la globalización. Revista de la CEPAL, (72), pp. 7-30.

Kolev, A. y J. E. Morales. (2005). La política monetaria y el sector informal. Banco Central de Venezuela. Colección economía y finanzas. Series de documentos de trabajo, (75), pp. $1-48$.

Koreshkova, T. (2006). A quantitative analysis on inflation as a tax on the underground economy. Journal of Monetary Economics, 53(4), pp. 773-796.

Leal J. C. (2013). Tax collection, the informal sector, and productivity. Review Economics Dynamics, 17(2), pp. 17-40.

Maloney, W. (1999). Does informality imply segmentation in urban labor markets? Evidence from sectorial transitions in Mexico. The World Bank Economic Review, 13(2), pp. 275-302.

Maloney, W. y J. Núñez. (2004). Measuring the impact of minimun wages. Evidence from Latin America. NBER Working Paper (9800), pp. 1-28.

Marcouiller, D., V. Ruiz de Castilla y C. Woodruff. (1997). Formal measures of the informal sector wage GAP in México, El Salvador y Perú. Economic Development and Cultural Change, 45(2), pp. 367-392.

Marjit, S., S. Kar y P. Sarkar. (2004). Capital mobility and informal wage in a small economy - two examples. South Asia Economic Journal, 5(2), pp. 261-266.

Martínez, D., J. Ávila, I. Caamal y F. García. (2009). Multiplicador del gasto público en México y el gasto en el sector agropecuario (1980-2001). Revista textual, (52), pp. 49-74.

Mayer-Foulkes, D. (2008). The human development trap in Mexico. World development, 36(5), pp. 775-796.

Montes-Rojas, G. y M. Santamaria. (2007). Source of productivity growth: evidence from the Mexican manufacturing sector. The North American Journal of Economics and Finance, 18(3), pp. 263-278.

Neck, R., M. Hofreither y F. Schneider. (1989). The consequences of progressive income taxation for the shadow economy: some theoretical considerations. The Political Economy of Progressive Taxation. Dieter Boes and Berhard Felderer, eds. Pp. 146-176.

Noyola, J. (2009). El desarrollo económico y la inflación en México y otros países latinoamericanos. Revista Clásicos, (3), pp. 161-177. 
96 Nueva Época REMEF (The Mexican Journal of Economics and Finance)

Núñez, J. (2002). Empleo informal y evasión fiscal en Colombia. Archivos de economía, Departamento Nacional de Planeación. Colombia.

Ochoa, S. (2002). Economía informal: evolución reciente y perspectivas. Centro de Estudios Sociales y de Opinión Pública. Cámara de diputados, LIX legislatura.

Pacheco, E. y S. Parker. (2001). Movilidad en el mercado de trabajo urbano: evidencias longitudinales para dos periodos de crisis en México. Revista Mexicana de Sociología, 63(2), pp. 3-26.

Parra, L. (2013). La empresarialidad informal como un reto de política económica: el caso de La Paz y El Alto, Bolivia. Estudios Gerenciales, (29), pp. 485-492.

Portes, A. (1995). En torno a la Informalidad: Ensayos sobre teoría y medición de la economía no regulada. $1^{\mathrm{a}}$ ed. Grupo editorial Miguel Ángel Porrúa. México D.F. Portes, A. y W. Haller. 2004. La economía informal. CEPAL, Series políticas sociales, (100), pp. 1-55.

Portes, A. y R. Schauffler. 1993. Competing perspectives on the Latin American informal sector. Population and Development Review, 19(1), pp. 33-60.

Ramos, A. L. (2015). Sector informal, economía informal e informalidad. Revista Iberoamericana para la Investigación y el Desarrollo Educativo, 6(11), pp. 1-12.

Rodríguez, G. I. y M. A. Calderón. (2015). La economía informal y el desempleo: el caso de la ciudad de Bucaramanga (Colombia). Revista Innovar Journal, 25(55), pp.41-58.

Samaniego, N. (2008). El crecimiento explosivo de la economía informal. Revista Economía, UNAM, 5(13), pp. 30-41.

Schneider, F. (2007). Shadow economies and corruption all over the world: new estimates for 145 countries. Economics, The open access, open assessment E-journal, (2007-9), pp. $1-47$.

Schneider, F. (2013). Work in the shadow1. Some facts. Economics working papers, Johannes Kepler University Linz, Austria.

Sheenan, C. y F. Riosmena. (2013). Migration, business formation, and the informal economy in urban Mexico. Social Science Research, 42(4). pp. 1092-1108.

Torgler, B. y F. Schneider. (2009). The impact of tax morale and institutional quality on the shadow economy. Journal of Economic Psychology, 30(2), pp. 228-245.

Tokman, V. (1982). Unequal development and the absorption of labour: Latin America 19501980. Cepal Review, (17), pp. 121-134.

Tokman, V. (2001). De la informalidad a la modernidad. Andros Impresores. Oficina Internacional del Trabajo, OIT.

Wallace, C. y R. Latcheva. (2006). Economic Transformation Outside the Law: Corruption, Trust in Public Institutions and the Informal Economy in Transition Countries of Central and Eastern Europe. Europe-Asia Estudies, 58(1), pp. 81-102. 
Revista Mexicana de Economía y Finanzas,Vol. 13 No.1, (2018), pp. 77-98 97

\section{Anexos}

Anexo 1. Empleo informal, ingreso tributario, INPC, inflación y salario mínimo en México.

\begin{tabular}{|c|c|c|c|c|c|}
\hline AÑo & $\begin{array}{c}\text { Empleo } \\
\text { informal } \\
\text { (Personas) }\end{array}$ & $\begin{array}{l}\text { Ingreso tributario } \\
\text { nominal (millones } \\
\text { de pesos) }\end{array}$ & INPC & $\begin{array}{c}\text { Inflación } \\
\text { (Porcentaje) }\end{array}$ & $\begin{array}{l}\text { Salario mínimo } \\
\text { (pesos) }\end{array}$ \\
\hline 1980 & $12,477,265$ & 485 & 0.09 & 29.8 & 0.14 \\
\hline 1981 & $14,437,091$ & 647 & 0.12 & 28.7 & 0.18 \\
\hline 1982 & $14,445,826$ & 967 & 0.23 & 98.8 & 0.24 \\
\hline 1983 & $13,935,836$ & 1,838 & 0.42 & 80.8 & 0.40 \\
\hline 1984 & $13,852,792$ & 3,049 & 0.68 & 59.2 & 0.60 \\
\hline 1985 & $13,822,292$ & 4,862 & 1.11 & 63.7 & 0.94 \\
\hline 1986 & $13,654,084$ & 8,979 & 2.28 & 105.7 & 2.48 \\
\hline 1987 & $13,106,507$ & 20,821 & 5.90 & 159.2 & 6.47 \\
\hline 1988 & $13,134,203$ & 47,385 & 8.94 & 51.7 & 7.25 \\
\hline 1989 & $12,404,855$ & 61,139 & 10.70 & 19.7 & 9.14 \\
\hline 1990 & $13,181,477$ & 79,317 & 13.91 & 29.9 & 10.79 \\
\hline 1991 & $13,372,460$ & 101,642 & 16.52 & 18.8 & 12.08 \\
\hline 1992 & $13,744,084$ & 126,764 & 18.50 & 11.9 & 12.08 \\
\hline 1993 & $14,043,332$ & 143,154 & 19.98 & 8.0 & 13.06 \\
\hline 1994 & $14,454,933$ & 160,318 & 21.39 & 7.1 & 13.97 \\
\hline 1995 & $14,234,996$ & 170,306 & 32.50 & 52.0 & 18.43 \\
\hline 1996 & $14,186,817$ & 226,006 & 41.50 & 27.7 & 24.30 \\
\hline 1997 & $14,412,399$ & 312,115 & 48.03 & 15.7 & 24.30 \\
\hline 1998 & $14,749,491$ & 404,225 & 56.96 & 18.6 & 31.91 \\
\hline 1999 & $14,498,704$ & 521,682 & 63.98 & 12.3 & 31.91 \\
\hline 2000 & $14,431,608$ & 581,703 & 69.71 & 9.0 & 35.12 \\
\hline 2001 & $14,327,323$ & 654,870 & 72.78 & 4.4 & 35.57 \\
\hline 2002 & $13,662,000$ & 728,284 & 76.93 & 5.7 & 39.74 \\
\hline 2003 & $13,588,598$ & 768,045 & 79.99 & 4.0 & 41.53 \\
\hline 2004 & $13,512,332$ & 769,386 & 84.14 & 5.2 & 43.30 \\
\hline 2005 & $13,415,794$ & 810,511 & 86.95 & 3.3 & 45.24 \\
\hline 2006 & $13,955,952$ & 890,078 & 90.47 & 4.1 & 47.05 \\
\hline 2007 & $14,043,101$ & $1,002,670$ & 93.87 & 3.8 & 48.88 \\
\hline 2008 & $14,754,481$ & 994,552 & 100.00 & 6.5 & 50.84 \\
\hline 2009 & $14,760,682$ & $1,129,553$ & 103.57 & 3.6 & 53.19 \\
\hline 2010 & $14,783,184$ & $1,260,459$ & 108.13 & 4.4 & 55.77 \\
\hline
\end{tabular}

Fuente: Elaborado con datos de INEGI, Banco de México, Secretaría de Hacienda y Crédito Público, Secretaría del trabajo, IMSS e ISSSTE. 
98 Nueva Época REMEF (The Mexican Journal of Economics and Finance)

Anexo 2. Tasa de desempleo, PIB real, PIB terciario real y base monetaria en México.

\begin{tabular}{|c|c|c|c|c|}
\hline AÑo & $\begin{array}{c}\text { Tasa de desempleo } \\
\text { abierto } \\
\text { (Porcentaje) }\end{array}$ & $\begin{array}{l}\text { PIB (Millones de } \\
\text { pesos de 2008) }\end{array}$ & $\begin{array}{c}\text { PIB terciario real } \\
\text { (Millones de pes os } \\
\text { de 2008) }\end{array}$ & $\begin{array}{c}\text { Base Monetaria } \\
\text { (Millones de } \\
\text { pesos) }\end{array}$ \\
\hline 1980 & 4.5 & $4,872,904,660$ & $2,927,865$ & 718 \\
\hline 1981 & 4.2 & $5,190,854,996$ & $3,163,286$ & 414 \\
\hline 1982 & 4.2 & $4,174,095,030$ & $2,601,797$ & 679 \\
\hline 1983 & 6.6 & $4,213,320,233$ & $2,502,006$ & 1,386 \\
\hline 1984 & 5.7 & $4,363,819,079$ & $2,581,311$ & 4,858 \\
\hline 1985 & 4.4 & $4,285,348,028$ & $2,516,831$ & 3,870 \\
\hline 1986 & 4.3 & $3,480,360,478$ & $2,022,941$ & 9,273 \\
\hline 1987 & 3.9 & $3,278,105,886$ & $1,859,879$ & 37,624 \\
\hline 1988 & 3.5 & $4,365,847,541$ & $2,631,223$ & 20,751 \\
\hline 1989 & 2.9 & $4,741,919,944$ & $2,986,749$ & 22,225 \\
\hline 1990 & 2.7 & $4,935,028,663$ & $3,153,620$ & 30,121 \\
\hline 1991 & 2.7 & $6,168,118,130$ & $3,453,941$ & 38,581 \\
\hline 1992 & 2.8 & $6,071,360,459$ & $3,755,932$ & 43,972 \\
\hline 1993 & 3.4 & $6,321,621,099$ & $3,994,199$ & 47,193 \\
\hline 1994 & 3.7 & $7,579,998,369$ & 4,306,919 & 56,935 \\
\hline 1995 & 6.2 & $6,856,582,710$ & $3,479,713$ & 66,809 \\
\hline 1996 & 5.5 & $6,746,874,228$ & $3,671,785$ & 83,991 \\
\hline 1997 & 5.4 & $7,057,526,599$ & $4,054,405$ & 108,891 \\
\hline 1998 & 4.7 & $7,113,421,276$ & $4,182,497$ & 131,528 \\
\hline 1999 & 3.7 & $7,568,443,564$ & $4,517,237$ & 188,718 \\
\hline 2000 & 3.4 & $7,342,894,534$ & $4,867,708$ & 208,943 \\
\hline 2001 & 3.6 & $7,584,655,777$ & $5,009,084$ & 225,580 \\
\hline 2002 & 3.9 & $7,894,472,899$ & $5,014,917$ & 263,937 \\
\hline 2003 & 4.6 & $8,612,495,634$ & $5,150,042$ & 303,614 \\
\hline 2004 & 5.3 & $8,838,833,827$ & $5,469,038$ & 340,178 \\
\hline 2005 & 4.7 & $9,595,764,555$ & $5,833,732$ & 380,034 \\
\hline 2006 & 4.6 & $9,956,091,010$ & $6,168,512$ & 449,821 \\
\hline 2007 & 4.8 & $10,369,878,316$ & $6,487,381$ & 494,744 \\
\hline 2008 & 4.9 & $9,522,558,037$ & $6,553,709$ & 577,543 \\
\hline 2009 & 6.6 & $9,928,316,605$ & $6,288,052$ & 632,032 \\
\hline 2010 & 6.4 & & $6,596,490$ & 693,423 \\
\hline
\end{tabular}

Fuente: Elaborado con datos de INEGI y Banco de México. 